\title{
effets de l'endiguement de la rive nord de l'estuaire de la Loire
}

par

\author{
R. Dupain
}

I. U.T. Génie civil, Saint-Nazaire

RESUME - Entre 1930 et 1979, le Port Autonome de Nantes St Nazaire a stabilisé le chenal de navigation sur la rive Nord de l'estuaire de la Loire en construisant une digue entre Donges et Montoir. Celle-ci a libéré des terrains qui anrès remblaiement ont été affectés à l'industrie lourde.

Ces terrains ont reçu pendant la construction (1930-1979) et du fait de celle-ci une couche de vase très plastique qui pose de sérieux problèmes de stabilité pour tous les remblais et stocks présents.

REMERCIEMENTS - Nous tenons à remercier le Port Autonome de Nantes St Nazaire et le BRGM pour tous les documents qui ont servi dans cette étude.

Les travaux d'aménagement de la Loire entre Nantes et St Nazaire ont eu pour but, depuis le l8ème siècle, d'améliorer les conditions de navigation en créant un chenal stable et d'entretien réduit. Ceux-ci se sont déroulés en trois phases essentielles :

- Endiguement de la section Nantes Le Pellerin (1859-1864)

- Doublement de la section intermédiaire Le Pellerin - Paimboeuf par un canal maritime (1869-1910), puis le canal s'étant révélé d'un gabarit tron faible, endiguement de la section intermédiaire

- Aménagement de la section aval Paimboeuf St Nazaire (à partir de 1933).

Création d'un chenal de navigation stable, suivant la rive Nord, d'où la construction par étapes (1933-1979) de la dique de Montoir entre Donges et St Nazaire. L'édification de cette digue a permis d'isoler des terrains qui ont été remblayés jusqu'à $7,50 \mathrm{~m}$ cote marine et qui accueillent aujourd'hui des équipements industriels et portuaires lourds.

C'est cette zone qui fait l'objet de notre étude (fig.1)

A St Nazaire o $\mathrm{NGF}=3,03 \mathrm{CM}-$

Niveau des plus hautes mers : 5,50 m CM.

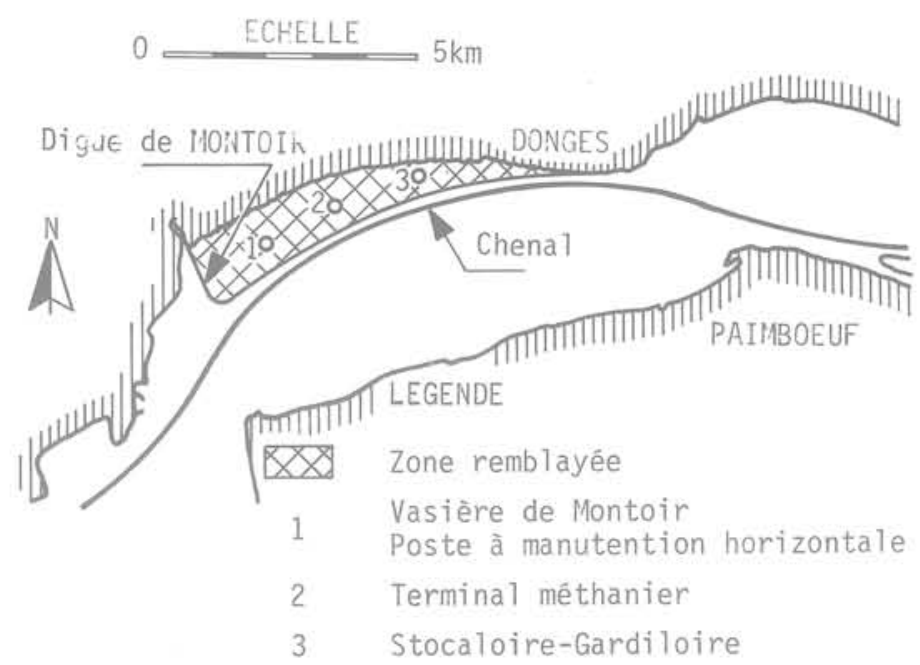

(Fig. $\mathrm{n}^{\circ} 1$ )

Nos travaux se sont dévelopnés selon trois directions :

- Evolution mornhologique de l'estuaire aval depuis 1927 (avant travaux) jusqu'en 1979 (fin des travaux).

- Compilation d'une centaine de sondages effectués dans la concavité de Montoir (zone remblayée entre la digue et l'ancien rivage du fleuve).

- Analyse de deux cas de rupture du sol dans la même zone. 

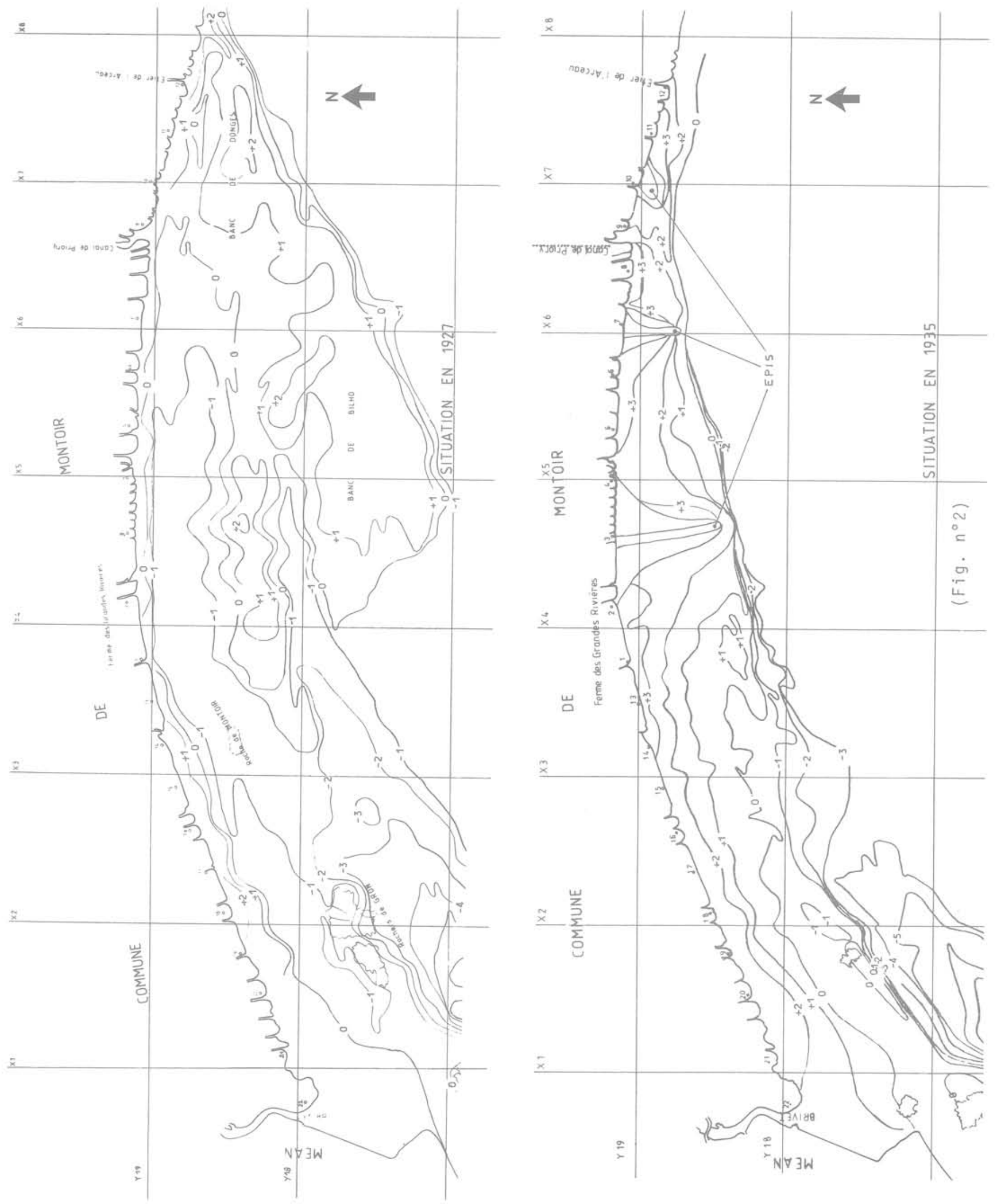

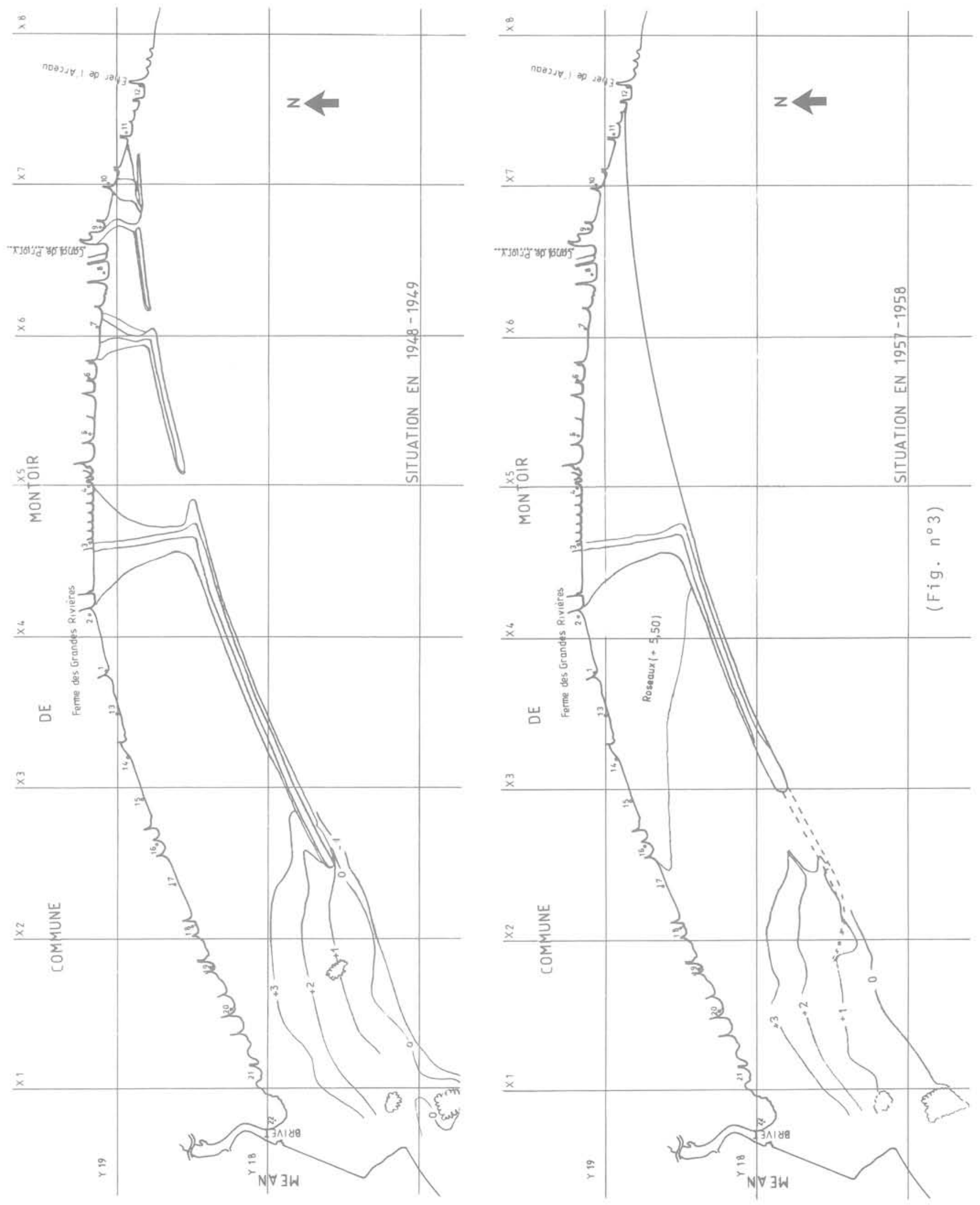
1.EVOLUTION DE L'ESTUAIRE AVAL DE 1927 à 1979 - ETUDE SUR CARTES. Réf.1

L'étude des cartes et levées bathymétriques effectués par le Port Autonome de Nantes St Nazaire entre 1927 et 1979 (11 cartes) nous a permis, d'une part, de reconstituer les différentes étapes de l'édification de la digue de Montoir et, d'autre part, de suivre l'évolution de l'envasement des zones ainsi abritées plus ou moins complétement de l'action des courants circulant dans 1 'estuaire.

Cette étude peut être résumée par les figures $n^{\circ} 2$ et 3 sur lesquelles sont représentées les phases d'évolution les plus marquantes.

Les étapes de la construction sont donc les suivantes :

- Construction de 3 épis perpendiculaires

à la rive (situation en 1935)

- Mise en place de la digue par tronçons

à partir des épis (situation en 1948-1949)

- Jonction des différents tronçons en une

digue continue (1957-1958) pour aboutir à

la fermeture de la concavité de Montoir en

1979 (Fig. n ${ }^{\circ}$ 1)

On peut constater que la sédimentation s'est effectuée, entre la digue et l'ancien rivage, pendant toute la durée de la construction et qu'elle a atteint une épaisseur de 3 à $6 \mathrm{~m}$ selon les secteurs.

Ces dépôts récents sont à comparer avec l'envasement qui a suivi la fermeture de l'ancien chenal rive gauche et qui a dépassé $5 \mathrm{~m}$ d'épaisseur.

Cette sédimentation vaseuse peut avoir diverses origines:

- La méthode de dragage avec redéposition d'une partie des matériaux dragués (dragage à l'américaine)

- La descente du bouchon vaseux en section aval lors des crues de la Loire

- Les apports de l'estuaire externe

Par ailleurs, une étude effectuée par le laboratoire central hydraulique de France (Réf.3) indique que la sédimentation a été permanente dans la zone aval de 1930 à nos jours, ceci n'excluant pas une érosion constante dans le chenal (autodragage).

En fait, l'endiguement et l'approfondissement du chenal ont eu pour conséquence une sédimentation argileuse importante dans les zones hors chenal et en particulier dans la concavité de Montoir.

Nous sommes donc en présence de dépôts superficiels qui ont atteint presque partout la côte $\simeq 5,50 \mathrm{~m} \mathrm{C.M} \mathrm{et} \mathrm{qui} \mathrm{ont} \mathrm{au}$ plus 45 ans d'âge. On peut donc présager d'un très mauvais comportement mécanique de ces sols fins.
2.ETUDE DES DOCUMENTS DE SONDAGES EFFECTUES DANS LA ZONE REMBLAYEE. Réf.2

Nous avons dépouillé les résultats d'une centaine de sondages exécutés au cours des 40 dernières années. Nous n'avons pu exploiter que 58 d'entre eux qui se situent géographiquement de la façon suivante : - 8 sondages dans la zone Ouest (vasière, poste à manutention horizontale, digue) . 32 sondages en partie centrale (terminal méthanier)

- 18 sondages en zone Est (entrepôts StocaLoire, usine d'engrais Gardiloire, quai à pondéreux, quai à liquides)

Nous avons reporté sur un graphique (fig.4) la position du toit et du mur de la couche d'argile molle superficielle pour chacun des sondages.

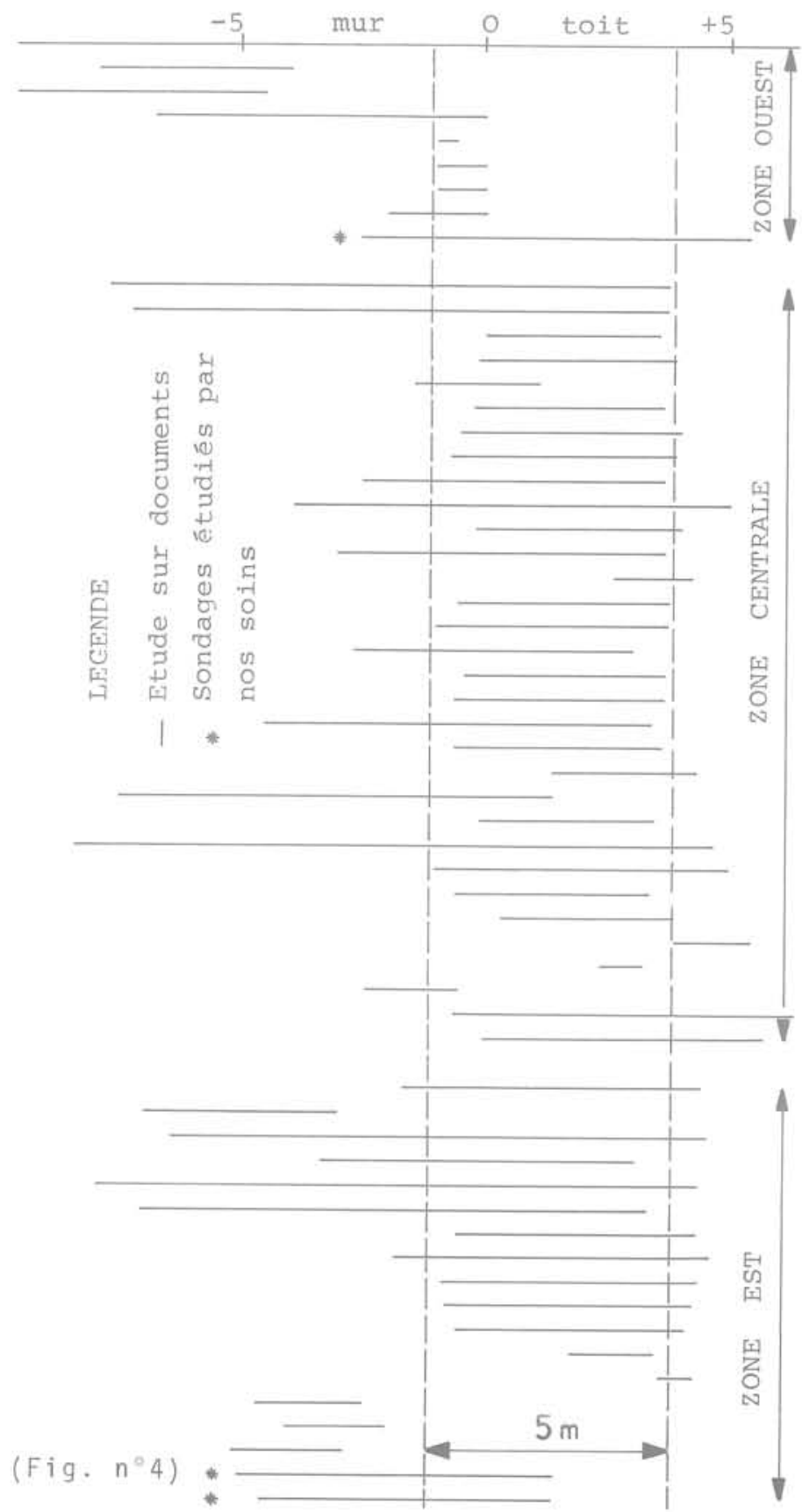


Ceci nous permet de confirmer l'existence d'une épaisseur d'argile molle superficielle d'environ $5 \mathrm{~m}$ avec un toit situé à environ $4 \mathrm{~m} \mathrm{CM}$ ce qui correspond aussi à la zone des hautes mers.

Les disparités constatées sur certains sondages ont diverses origines :

- Difficulté d'interprétation du langage du sondeur et des documents correspondants - Sondages concernant les ouvrages portuaires qui correspondent à des sites immergés (quais)

Le dépouillement statistique des résultats nous donne une épaisseur moyenne de la couche d'argile molle superficielle de $5 \mathrm{~m}$ avec un écart type de $3 \mathrm{~m}$.

Il y a donc bon accord entre ce chiffre et celui issue de l'étude sur cartes.

\section{ETUDE DE DEUX CAS DE RUPTURE DU SOL} APRES CHARGEMENT .

Deux cas de rupture de sol après chargement ont été étudiés :

- Route d'accès au terminal roulier poste à manutention horizontale avec franchissement de la vasière Ouest de Montoir.

- Aire de stockage de phosphates de l'entreprise Stocaloire.

\section{ler cas :}

Le franchissement de la vasière ouest de Montoir a été réalisé par une route sur remblai hydraulique depuis la digue bordant la Loire au Sud vers l'ancien rivage au Nord. Il y a eu rupture généralisée de la vase superficielle par le remblai hydraulique.

Un sondage effectué à proximité de la route a donné la coupe suivante :

$5,40 \mathrm{~m} \mathrm{CM}$ à $1,40 \mathrm{~m} \mathrm{CM}$

Vase très fluide

$1,40 \mathrm{~m} \mathrm{CM}$ à $-2,60 \mathrm{~m} \mathrm{CM}$

Argile très plastique

$-2,60 \mathrm{~m} \mathrm{CM}$ à $-12,90 \mathrm{~m} \mathrm{CM}$

Alternance d'argile plastique et d'argile peu plastique à fins lits de sable (apnelée "jalle" localement)

$-12,90 \mathrm{~m} \mathrm{CM}$ à $-13,20 \mathrm{~m} \mathrm{CM}$

Sables coquillers

L'étude des sols a montré que nous sommes en présence d'argiles vierge pour la première couche et d'argiles sous consolidées à normalement consolidées pour les couches inférieures.

De plus, la mesure de la cohésion non drainée $\mathrm{Cu}$ en place au scissomètre de chantier, a montré que entre 5,40 et $1,60 \mathrm{~m} \mathrm{CM}$ les valeurs de cohésion intacte et remaniée sont identiques. C'est donc que cette couche a été poinçonné par le remblai. on constate aussi que cette même couche est celle qui s'est dénosée du fait de la construction de la digue (Fig. 2 et 3 ).

\section{2ème cas:}

L'édification d'un stock de $10 \mathrm{~m}$ de haut de phosnhates $(\gamma=14 \mathrm{kN} / \mathrm{m} 3)$ a provoqué le poinçonnement du sol avec formation de bourrelets latéraux. Nous avons effectué deux sondages avec prélèvement d'échantillons intact en continu l'un à l'emplacement même du stock, l'autre dans une zone intacte voisine mais hors de l'influence de celuici.

La coune du sol est la suivante :

$7,50 \mathrm{~m} \mathrm{CM}$ à $\quad 3,50 \mathrm{~m} \mathrm{CM}$

Remblai hydraulique

$3,50 \mathrm{~m} \mathrm{CM}$ à $-3 \mathrm{~m} \mathrm{CM}$

Argile très plastique

$-3 \mathrm{~m} \mathrm{CM}$ à $-16 \mathrm{~m} \mathrm{CM}$

Alternance d'argile nlastique à peu plastique (jalle) et de passages sableux plus ou moins épais.

L'étude détaillée à nartir notamment de 22 essais oedométriques a conduit aux conclusions suivantes :

- Nous sommes en présence dans les deux cas d'argiles vierges de $3,50 \mathrm{~m}$ à - $10 \mathrm{~m} \mathrm{CM}$ et d'argiles sous consolidées à vierges même aux plus grandes profondeurs. Les seules couches normalement consolidées sont celles d'argiles à fins lits de sable (jalle) - La mise en coIrcidence des coupes verticales de sol pour les deux sondages, rendue nossible par la présence d'une couche d'huîtres à $-15,50 \mathrm{~m} \mathrm{CM}$, montre le fluace de 0,50 à $1 \mathrm{~m}$ d'argile dans la couche sunérieure ce qui a donné naissance aux bourrelets latéraux.

- La présence du stockage pendant 4 ans n'a pas provocué de consolidation anoréciable des sols (essais à l'Oedomètre)

Ceci met par conséquent en évidence le mauvais comportement de la couche d'argile superficielle.

\section{CONCLUSION}

La stabilisation du chenal de navigation sur la rive droite de l'estuaire de la Loire entre Donges et St Nazaire, a conduit à l'édification de la dique de Montoir. Cet ouvrage dont la finalité a été atteinte a eu pour conséquence un dénôt de vase très plastique d'environ $5 \mathrm{~m}$ d'énaisseur dans la concavité de Montoir. 
Ainsi, les terrains mis hors d'eau du fait de cette construction ont ils été recouverts d'un sédiment de caractéristiques mécaniques très mauvaises. Ceci a conduit à des difficultés importantes lors de la mise en place de remblais ou de stocks destinés au fonctionnement des industries implantées dans la concavité de Montoir.

La construction de la digue de Montoir a donc conduit à la modification des propriétés mécaniques du sol en place ce qui a rendu trềs difficile toutes les mises en place de remblais.

Cet aspect négatif était prévisible mais on peut dire aussi que la création de zones industrielles n'était pas le but recherché au début du projet en 1930.

\section{Références :}

- Levés de l'estuaire de la Loire -PANSN - Documents dénosés au BRGM - Nantes

- LCHF : Loire Maritime - Aménagement de I'estuaire. 